\title{
Correction to: Multiple origin of flightlessness in Phaneropterinae bushcrickets and redefinition of the tribus Odonturini (Orthoptera: Tettigonioidea: Phaneropteridae)
}

\author{
Beata Grzywacz ${ }^{1}$ - Arne W. Lehmann ${ }^{2}$ - Dragan P. Chobanov ${ }^{3} \cdot$ Gerlind U. C. Lehmann ${ }^{4}$
}

Published online: 16 July 2018

(C) Gesellschaft für Biologische Systematik 2018

\section{Correction to: Organisms Diversity \& Evolution https://doi.org/10.1007/s13127-018-0370-x}

The data in the Table 1 of the original version of this article were inadvertently shifted during the editing process. Therefore the entries as published in this table did not correspond to their correct data.

The published version of the Table 1 has now been corrected in this article. The columns such that all the previously misaligned data do now properly correspond to their correct respective rows as were present in the authors' final submitted manuscript version.

The original article has been corrected.

The online version of the original article can be found at https://doi.org/ 10.1007/s13127-018-0370-x

Beata Grzywacz

grzywacz@isez.pan.krakow.pl

1 Institute of Systematics and Evolution of Animals, Polish Academy of Sciences, Sławkowska 17, 31-016 Krakow, Poland

2 Friedensallee 37, 14532 Stahnsdorf, Germany

3 Institute of Biodiversity and Ecosystem Research, Bulgarian Academy of Sciences, 1 Tsar Osvoboditel Boul, 1000 Sofia, Bulgaria

4 Evolutionary Ecology, Department of Biology, Humboldt University Berlin, Invalidenstrasse 110, 10115 Berlin, Germany 


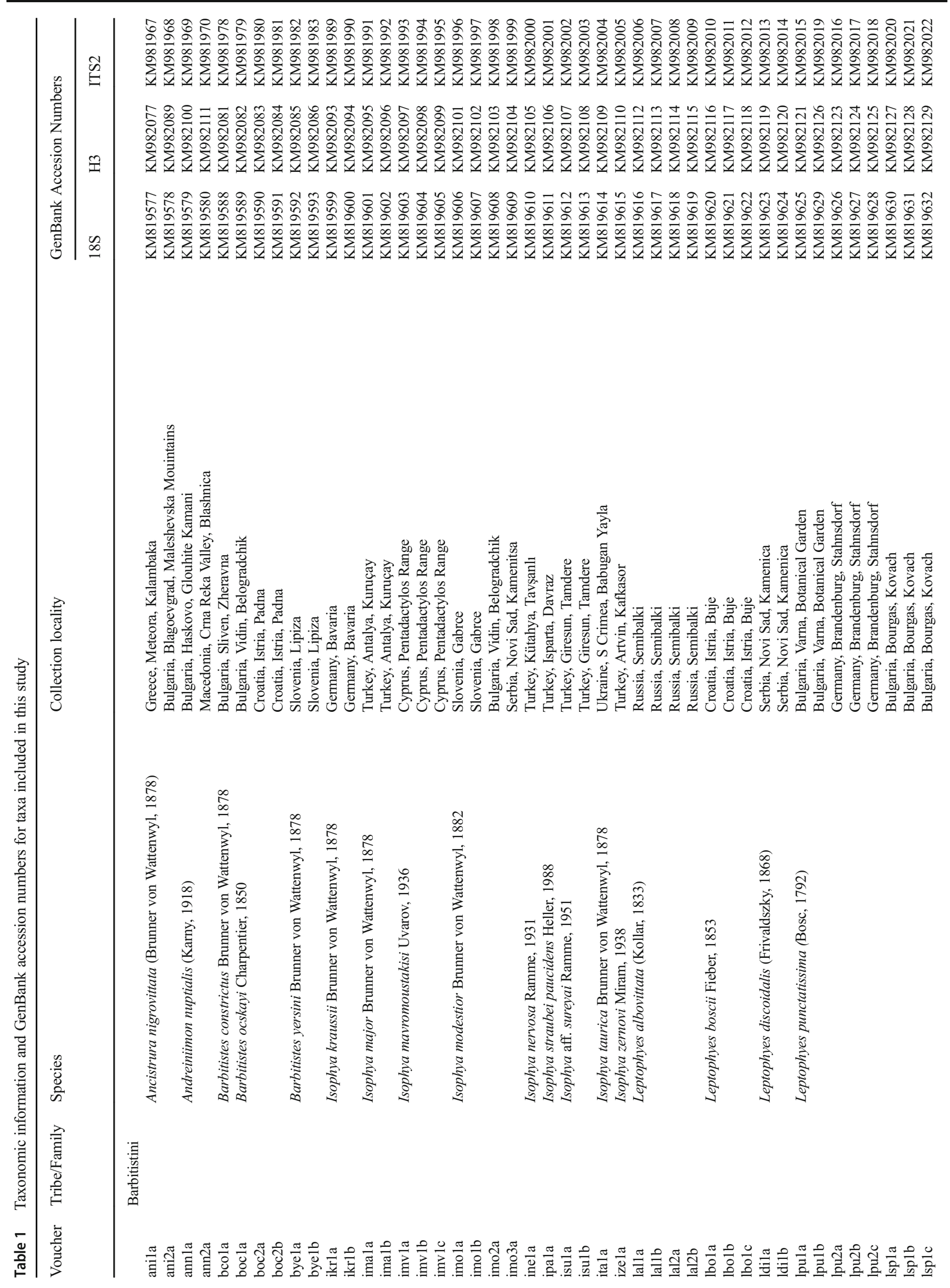




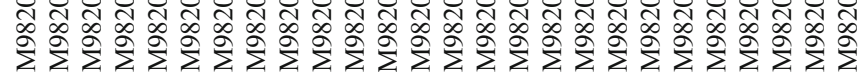

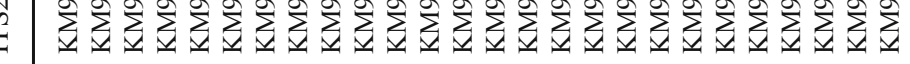

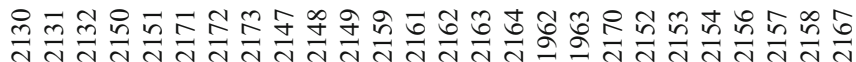

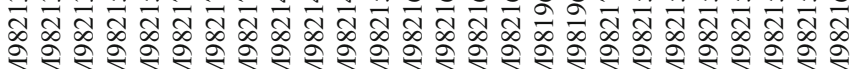

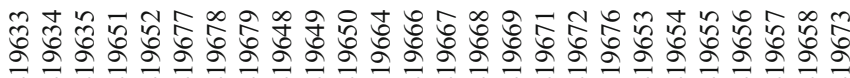
$\sum^{\infty} \sum^{\infty} \sum^{\infty} \sum^{\infty} \sum^{\infty} \sum^{\infty} \sum^{\infty} \sum^{\infty} \sum^{\infty} \sum^{\infty} \sum^{\infty} \sum^{\infty} \sum^{\infty} \sum^{\infty} \sum^{\infty} \sum^{\infty} \sum^{\infty} \sum^{\infty} \sum^{\infty} \sum^{\infty} \sum^{\infty} \sum^{\infty} \sum^{\infty} \sum^{\infty} \sum^{\infty} \sum^{\infty} \sum^{\infty} \sum^{\infty}$

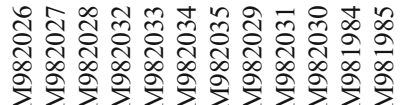

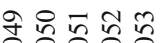

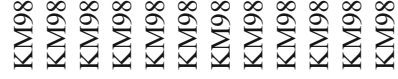

每

$\sum_{i} \sum_{i}^{\infty} \sum_{i=1}^{\infty}$

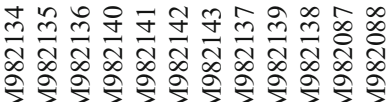

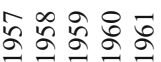

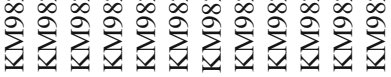

$\sum_{\underline{1}}^{\infty} \sum_{\underline{1}}^{\infty} \sum_{\underline{\Sigma}}^{\infty} \sum_{\underline{\Sigma}}^{\infty}$

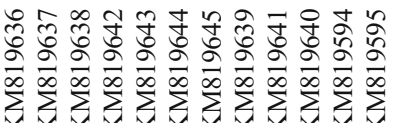

ڤิ:

$\sum_{\Sigma}^{\infty} \sum_{\Sigma}^{\infty} \sum_{\Sigma}^{\infty} \sum_{\Sigma}^{\infty}$

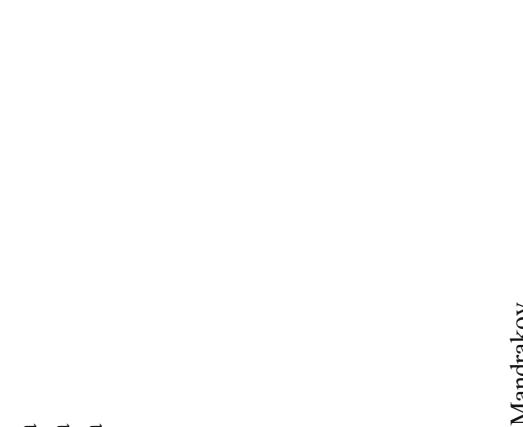

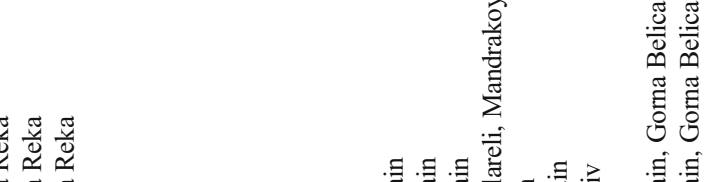

到

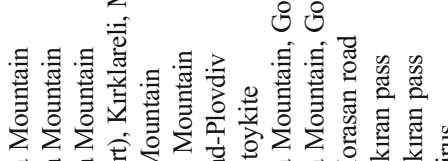

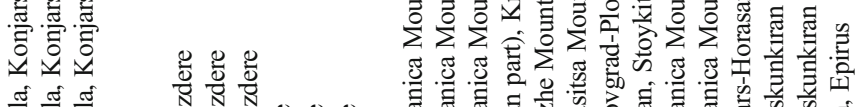

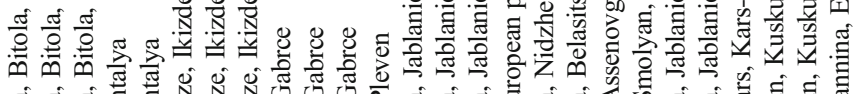

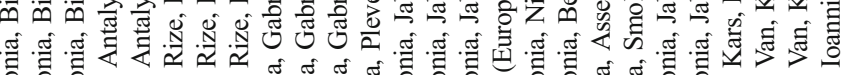

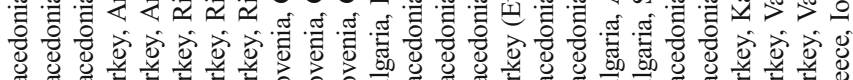

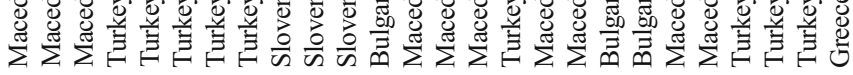

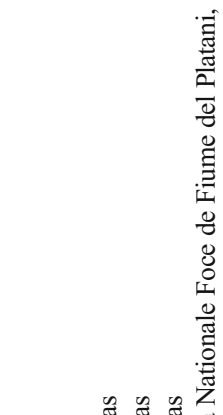

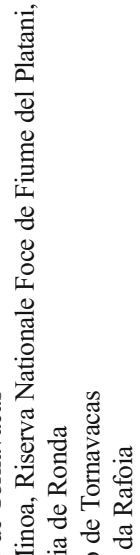

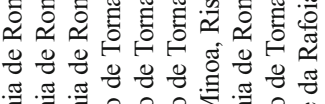

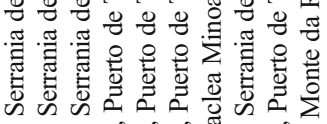

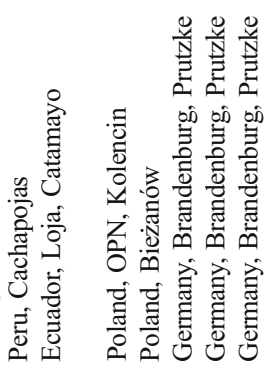

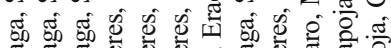

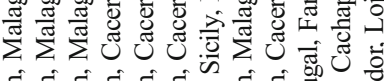

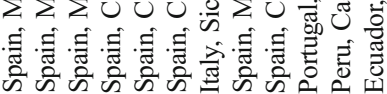

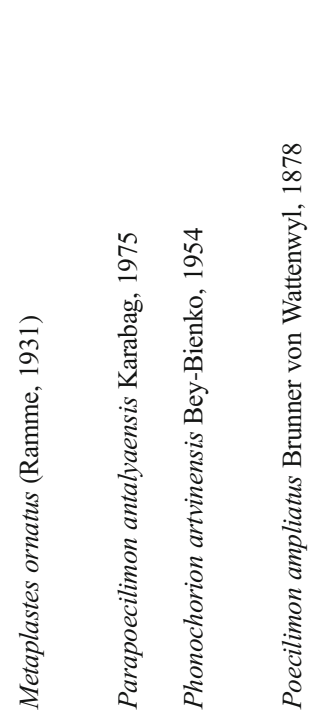

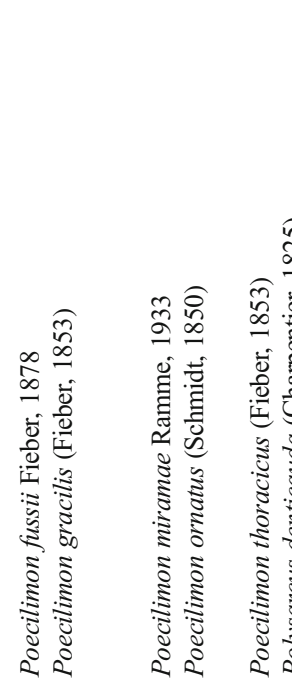

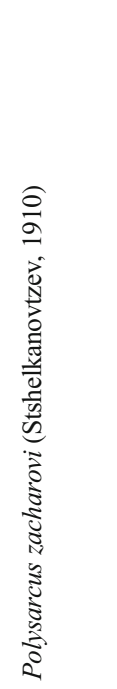

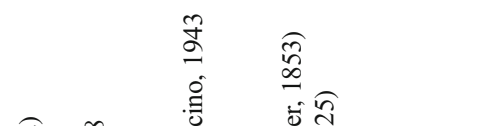




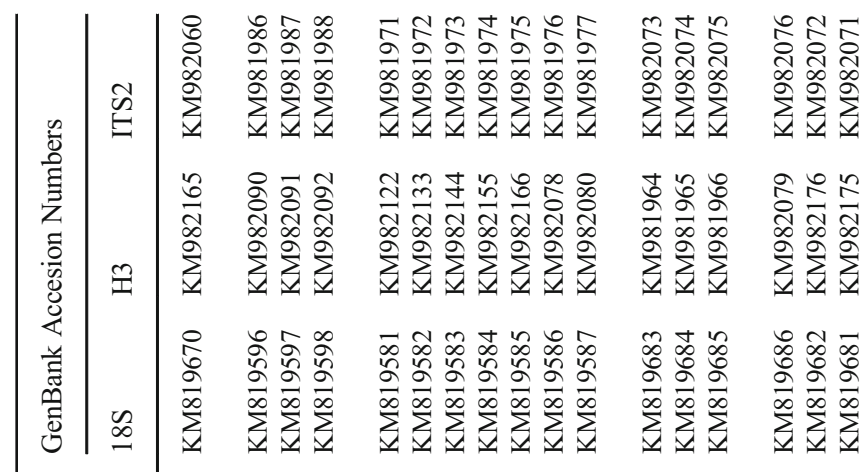

总怘

悉等

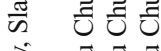

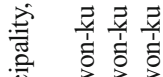

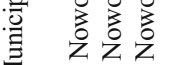

范

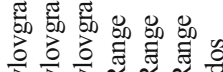

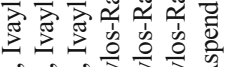

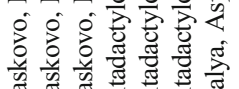

콩

总总总

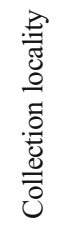

总

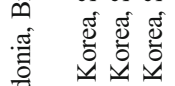

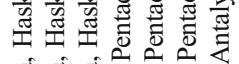

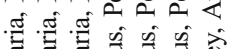

뜰 을

응

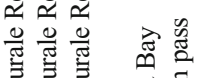

究 圠

造总

○े

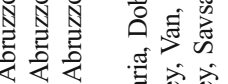

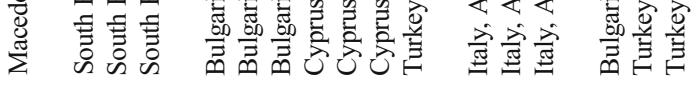

.

intor.

(1)

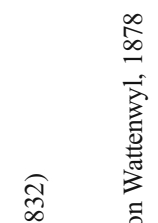<smiles>CCCCCCC</smiles>

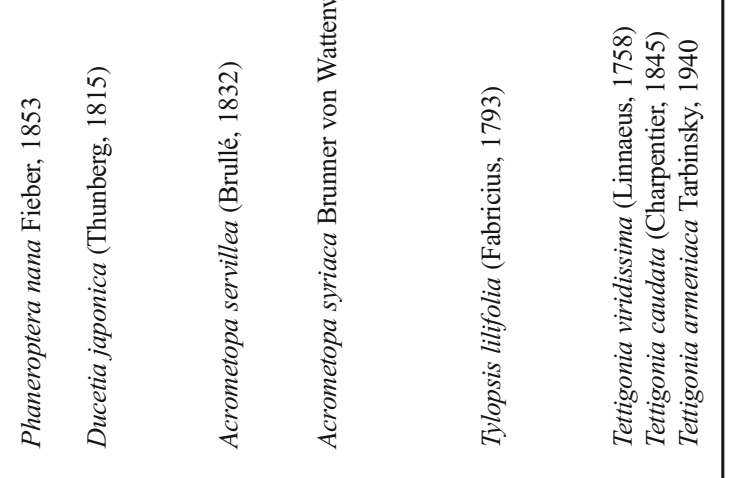

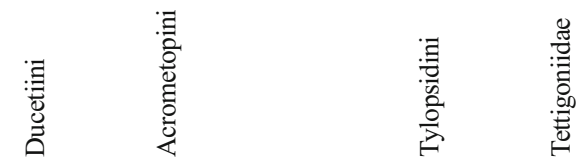

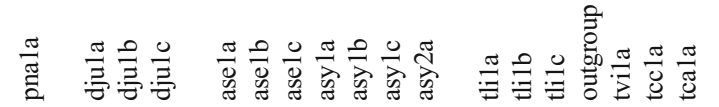

